\title{
Nuevos antecedentes en la distribución y ecología de Botrychium dusenii (H. Christ.) Alston (Ophioglossaceae) asociado a ambientes antropogénicos de la provincia de Coyhaique, Región de Aysén, Chile
}

\author{
New antecedents on the distribution and ecology of Botrychium dusenii (H. Christ.) \\ Alston (Ophioglossaceae) associated with anthropogenic environments in the Coyhaique \\ province, Aysén Region, Chile
}

\author{
Pablo Bravo-Monasterio ${ }^{1,2}$, Patricio Peñailillo ${ }^{3}$ \& Alex Fajardo ${ }^{4}$ \\ ${ }^{1}$ Facultad de Ciencias Forestales, Universidad de Talca, 2 norte 685, casilla 747, Talca, Chile. \\ 2Dirección Actual: Laboratorio de Invasiones Biologicas (LIB), Facultad de Ciencias Forestales, Universidad de Concepción, \\ Casilla 160-C, Concepción, Chile. \\ ${ }^{3}$ Instituto de Biología Vegetal y Biotecnología, Universidad de Talca, 2 norte 685, Casilla 747, Talca, Chile. \\ ${ }^{4}$ Centro de Investigación en Ecosistemas de la Patagonia (CIEP), Conicyt-Regional R10C1003, Ignacio Serrano 509, \\ Coyhaique, Chile. \\ pabravo@utalca.cl
}

\begin{abstract}
Conducting botanical and ecological explorations with the objective of studying the floristic composition of post-fire communities in the Aysén Region, we found new regional records of the fern Botrychium dusenii (H. Christ.) Alston (Ophioglossaceae). These findings, that extend the north limit of the species distribution, occurred in open areas affected by historical human-provoked fires (happening between 1950 and 1960) during the regional process of colonization.
\end{abstract}

Según Rodríguez (1995), en Chile Botrychium dusenii (H. Christ.) Alston se distribuye sólo en la Región de Magallanes, entre las provincias de Última Esperanza (50³0’S) y Antárctica Chilena (5455'S). En estas latitudes la ocurrencia de $B$. dusenii ha sido frecuentemente documentada por diversos autores (Pisano 1971, Moore 1974, Pisano 1980, Dollenz 1982, Arroyo et al. 1992, Henríquez et al. 1995, Moore \& Pisano 1997, De la Sota et al. 1998, Domínguez et al. 1999, Henríquez 2002, Rodríguez et al. 2009). Sin embargo, para la Región de Aysén el escenario es distinto debido a la existencia de sólo una cita correspondiente a la localidad de Chile Chico, en la Reserva Nacional Lago Jeinimeni, en la provincia de General Carrera (4650'58.19'S - 72¹'32.03'W), como parte de la flora acompañante de Valeriana moyanoi Speg. (Saldivia \& Rojas 2006). Anteriormente existe una cita de De Lichtenstein (1944), donde aparece "Territorio de Aisén: Lago San Martín, Donat 503, 18-II-1932, (SI; Herb. Looser)" como lugar de recolecta, aunque hay un error, ya que el Lago San Martín se encuentra en territorio Argentino.

En el mapa de distribución presentado por Rodríguez et al. (2009) para esta especie, no se registra $B$. dusenii para la Región de Aysén, pero sí se menciona por De la Sota y
Ponce (2008) y Domínguez et al. (2011). Para corroborar la presencia de $B$. dusenii tanto en la Región de Magallanes, como la de Aysén, se revisaron los ejemplares de los herbarios del Departamento de Botánica de la Universidad de Concepción (CONC, N=17) y del Museo de Historia Natural ( $\mathrm{SGO}, \mathrm{N}=8$ ). Los registros para esta última región corresponden a los ejemplares de herbario número CONC 169202, SGO 153341, SGO 100235, SGO 100249, SGO 106201 y SGO 85453, los cuales geográficamente incluyen las provincias de Capitán Prat, General Carrera y Coyhaique. En este estudio documentamos una nueva localidad para $B$. dusenii en la Provincia de Coyhaique ubicada a $1 \mathrm{~km}$ del camino al sector Mano Negra y a 3 $\mathrm{km}$ del cruce entre la ruta 7 y el desvío a Mano Negra

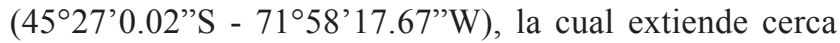
de $520 \mathrm{~km}$ y más de cinco grados de latitud el límite norte anteriormente establecido en los $50^{\circ} 30^{\prime} \mathrm{S}$ por Rodríguez (1995) y Rodríguez et al. (2009).

También hemos observado en terreno la presencia de $B$. dusenii en otras localidades de la Región de Aysén (aunque más al sur que nuestro hallazgo más septentrional): Reserva Nacional Cerro Castillo ubicada en Provincia de

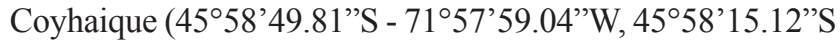




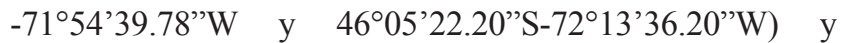
Provincia General Carrera, Valle del Río Cacho, Cordón Contreras (46 58'13,5'S - 7305'10,4”W) (Simón Pfanzelt com. pers.). Utilizando toda esta información se confeccionó un mapa de distribución de $B$. dusenii para la especie en la Región de Aysén (Fig. 1).

Llama la atención el escaso número de ejemplares en los herbarios estudiados $(\mathrm{N}=25)$, los autores de esta nota piensan que esta escasez de recolectas en la región, se puede deber a: 1) la falta de exploraciones botánicas; 2) el tamaño reducido de la especie, $\leq 10 \mathrm{~cm}$ de altura, el cual la hace casi indistinguible de la vegetación circundante (Fig. 2 A y B); y 3) la corta permanencia de la parte aérea del esporofito durante su ciclo anual de crecimiento.

Este helecho está catalogado dentro de los endemismos del centro de diversidad templado-subantártico (Ponce et al. 2002). Este centro de diversidad está asociado a los bosques de la Patagonia Austral y Tierra del Fuego y ha sido descrito fitogeográficamente por Pisano (1997) como Fuego-Patagonia Austral, el cual se ubica aproximadamente entre los $46^{\circ} \mathrm{S}$ (Chile Chico) y los $47^{\circ} \mathrm{S}$ (Golfo de Penas) llegando hasta los $56^{\circ} \mathrm{S}$ (Isla Hornos, en el archipiélago homónimo).

En la parte más meridional de Fuego-Patagonia Austral (Tierra del Fuego), B. dusenii estaría asociado a tres zonas vegetacionales que corresponden a los Llanos Fuego-patagónicos-pastos, bosque caducifolio y pantanos de Sphagnum y al bosque siempreverde (Moore 1974); y recientemente en el extremo pluviométrico árido hacia el oriente en la estepa patagónica (Domínguez et al. 2011). En cambio, en su parte más septentrional (Provincia de Coyhaique), B. dusenii estaría restringido al bosque denominado por Pisano (1981) como Bosque Magallánico deciduo con especies andino-patagónicas, probablemente el límite más boreal de $B$. dusenii.

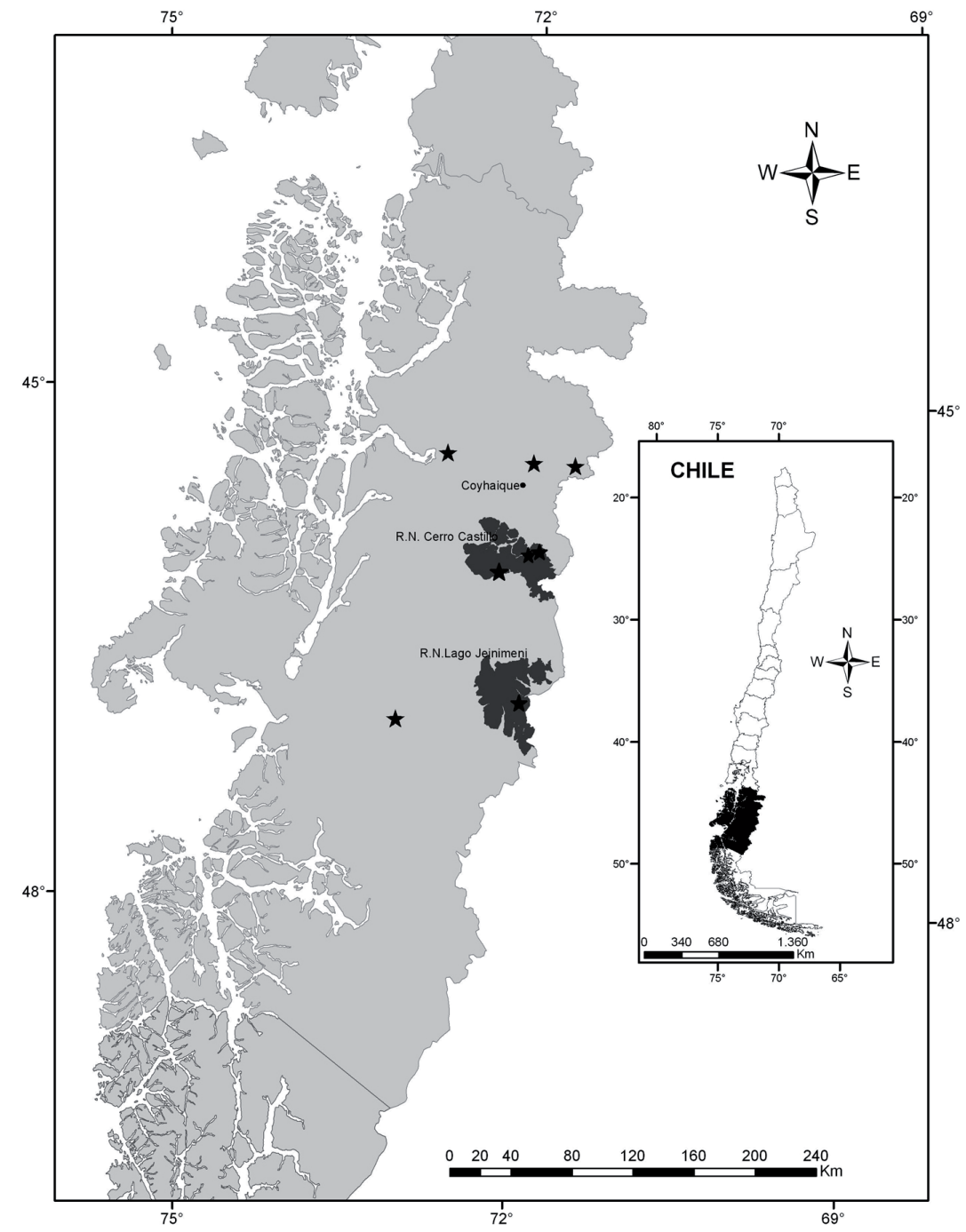

Figura 1. Distribución de Botrychium dusenii en la Región de Aysén, Chile. Las estrellas indican la ubicación de la especie en la Región de Aysén.

FIgURE 1. Distribution of Botrychium dusenii in Aysén Region, Chile. Stars stand for locations in Aysén Region. 

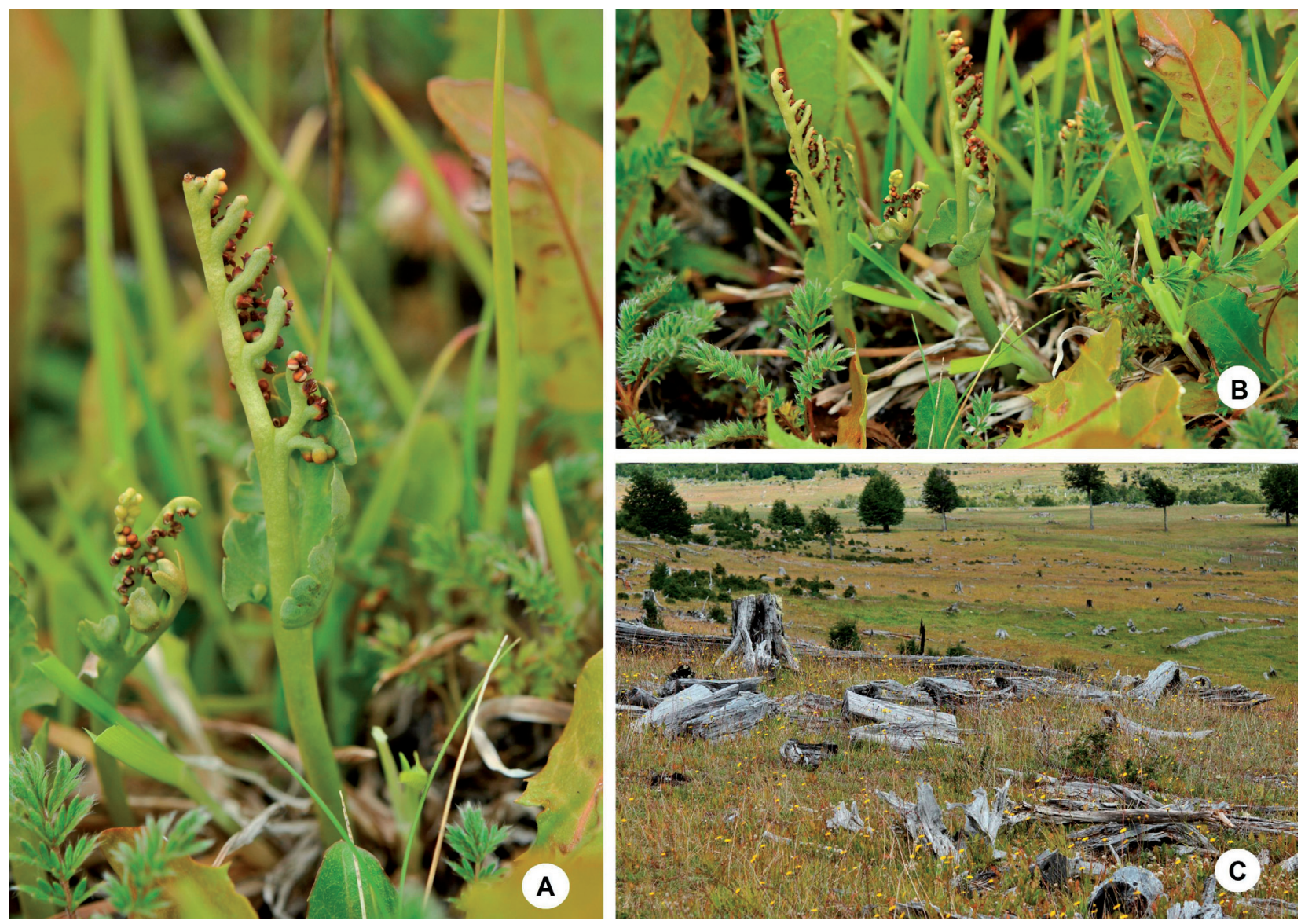

Figura 2. Botrychium dusenii: A. Planta, B. Individuos en praderas abiertas dominadas por un estrato herbáceo, C. Hábitat en su límite norte.

Figure 2. Botrychium dusenii: A. The fern, B. Specimens growing in open prairies dominated by herbaceous species, C. Habitat in its northern limit.

Al revisar los antecedentes del hábitat de $B$. dusenii proporcionados por la literatura y los registros de herbarios, se encuentran ciertas generalidades tales como, que se presenta en suelos arenosos y pedregosos, sitios expuestos en el piso subalpino, turberas de Sphagnum, praderas y estepas (Pisano 1980, Arroyo et al. 1992, De la Sota 1998). Según nuestras observaciones, $B$. dusenii se encuentra en zonas de ecotono de bosque degradado y matorral subandino y en praderas abiertas con presencia de especies introducidas (e.g. Holcus lanatus L., Poa annua L., Rumex acetosella L. y Trifolium repens L.) (Fig. 2C). Los hábitats descritos anteriormente son muy similares a aquéllos de otras especies de Botrychium, e.g., Botrychium multifidum (Gmel.) Rupr., una especie del hemisferio norte que se encuentra a menudo en praderas y sitios abiertos en las zonas boscosas, comúnmente asociada a Pinus contorta Douglas \& Loudon (Anderson 2005). Esta información, más lo propuesto por Wagner (1990), señalan que las especies del género Botrychium serían plantas adaptadas a las perturbaciones. Esto hace suponer que los cambios florísticos provocados por los incendios forestales de décadas anteriores en la Región de Aysén dieron origen a comunidades ruderales o artificiales asociadas a suelos degradados (Pisano 1997, Quintanilla 2005, Quintanilla et al. 2008), ambientes que podrían haber favorecido el desarrollo y establecimiento de nuevas poblaciones de $B$. dusenii.

Finalmente, en términos de conservación, $B$. dusenii ha sido señalado como una especie Rara (Rodríguez 1995, Baeza et al. 1998) debido, principalmente, a su distribución restringida y a su escasa densidad (Baeza et al. 1998) y posteriormente Vulnerable por Rodríguez et al. (2009). En la Región de Aysén esta especie estaría bien representada en las áreas protegidas por el estado (SNASPE), Reserva Nacional Cerro Castillo y Reserva Nacional Lago Jeinimeni.

Material estudiado: CHILE, XI Región de Aysén, Prov. de Coyhaique, comuna de Coyhaique, a $1 \mathrm{~km}$ del camino al sector Mano Negra y a $3 \mathrm{~km}$ del cruce entre la ruta $7 \mathrm{y}$

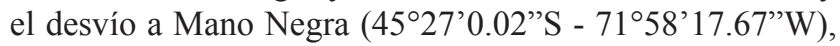
elevación $828 \mathrm{~m}$, en pradera abierta con suelo aparentemente degradado (erosión visible), cerca del límite con bosque secundario de lenga y plantación de Pinus contorta, Bravo \& Fajardo s/n, 24/02/2011 (CONC 173183). 


\section{AGRADECIMIENTOS}

Al señor Simón Pfanzelt por facilitar valiosa información de terreno sobre esta especie y a las curadoras del Herbario Gloria Rojas (SGO) y Alicia Marticorena (CONC) por las facilidades otorgadas en este estudio. Además, se agradece a los revisores por sus sugerencias en el manuscrito. Este trabajo se realizó en el marco del proyecto Fortalecimiento SS-2008-10 del Centro de Investigación en Ecosistemas de la Patagonia (CIEP).

\section{BIBLIOGRAFÍA}

Anderson, D.G. 2005. Botrychium multifidum (Gmel.) Rupr. (leathery grapefern): a technical conservation assessment. [Online]. USDA Forest Service, Rocky Mountain Region. Available: http://www.fs.fed.us/r2/projects/scp/ assessments /botrychiummultifidum.pdf [date of access].

Arroyo, M.T.K., C. von Bohlen, L. Cavieres \& C. Marticorena. 1992. Survey of the alpine flora of Torres del Paine National Park, Chile. Gayana Botánica 49: 47-70.

Baeza, M., E. Barrera, J. Flores, C. Ramírez \& R. Rodríguez. 1998. Categorías de conservación de Pteridophyta nativas de Chile. Boletín del Museo Nacional de Historia Natural 47: 23-46.

Dollenz, O. 1982. Estudios fitosociológicos en el Archipiélago de Cabo de Hornos. III. Relevamientos en la isla Deceit. Anales del Instituto de la Patagonia 13: 145-151.

De la Sota, E.R. \& M.M. Ponce. 2008. Ophioglossaceae. En: F.O. Zuloaga, O. Morrone \& M.J. Belgrano (eds.), Catálogo de las plantas vasculares del Cono Sur (Argentina, Sur de Brasil, Chile, Paraguay y Uruguay). Vol. 1, pp. 99-101. Missouri Botanical Garden e Iboda, Conicit. St. Louis, Missouri, USA.

De la Sota, E. R., M.M. Ponce, M. A. Morbelli \& L. Cassá de Pazos. 1998. Pteridophyta. En: M.N. Correa (ed.), Flora Patagónica: Vol. 8, pp. 282-369, Buenos Aires, Argentina.

Domínguez, E., E. Pisano \& O. Dollenz. 1999. Colonización Vegetal en el Glaciar Nueva Zelandia, Cordillera Darwin Chile. Anales del Instituto de la Patagonia, Serie Ciencias Naturales 27: 7-16.

Domínguez, E., A. SuÁrez, N. Bahamonde \& S. Opazo. 2011. Botrychium dusenii (H.Christ) Alston (Ophioglossaceae) nueva adición para la flora vascular del Parque Nacional Pali Aike, Región de Magallanes, Chile. Gayana Botánica 68(2): 345-349.

Henríquez, J.M., E. Pisano \& C. Marticorena. 1995. Catálogo de la flora vascular de Magallanes (XII Región), Chile. Anales del Instituto de la Patagonia, Serie Ciencias Naturales 23:
$5-30$.

Henríquez, J.M. 2002. Análisis de la flora vascular de valles glaciares de la Región de Magallanes, Chile. Anales del Instituto de la Patagonia, Serie Ciencias Naturales 30: 2540.

Lichtenstein, J.S. 1944. Las Ofioglosáceas de la Argentina, Chile y Uruguay. Darwiniana 6(3): 380-441.

Moore, D.M. 1974. Catálogo de las plantas vasculares nativas de Tierra del Fuego. Anales del Instituto de la Patagonia 5(12): $105-121$

Moore, D. \& E. Pisano. 1997. Biotic Colonization of recently deglaciated areas in fuego-patagonia -phytogeographical considerations. Anales del Instituto de la Patagonia, Serie Ciencias Naturales 25: 21-46.

Pisano, E. 1971. Comunidades vegetales del área del Fiordo Parry, Tierra del Fuego, Anales del Instituto de la Patagonia 2(12): 93-133.

PisAno, E. 1980. Catálogo de la flora vascular del archipiélago de Cabo de Hornos, Anales del Instituto de la Patagonia 11: 151-189.

Pisano, E. 1981. Bosquejo fitogeográfico de fuegopatagonia. Anales del Instituto de la Patagonia 12: 159-171.

Pisano, E. 1997. Los bosques de Patagonia Austral y Tierra del Fuego Chilenas. Anales del Instituto de la Patagonia, Serie Ciencias Naturales 25: 9-19.

Ponce, M., K. Mehltrenter \& E. DE la Sota. 2002. Análisis biogeográfico de la diversidad pteridofítica en Argentina y Chile Continental. Revista Chilena de Historia Natural 75: 703-717.

Quintanilla, V. 2005. Estado de recuperación del bosque nativo en una cuenca nordpatagónica de Chile, perturbada por grandes fuegos acaecidos 50 años atrás $\left(44^{\circ}-45^{\circ} \mathrm{S}\right)$. Revista de Geografía Norte Grande 34: 73-92.

Quintanilla, V., G. Meaza \& M. J. Cuesta. 2008. Perturbaciones actuales del bosque norpatagónico chileno derivadas de los efectos de grandes fuegos de medio siglo atrás. Estudio preliminar en la cuenca andina del río Figueroa. Boletín de la AGE 47: 109-124.

Rodríguez, R. 1995. Pteridophyta. En: C. Marticorena \& R. Rodríguez (eds.), Flora de Chile, Vol. 1, pp. 119-309. Ediciones Universidad de Concepción, Concepción, Chile.

Rodríguez, R., D. Alarcón \& J. Espejo. 2009. Helechos Nativos del Centro y Sur de Chile. Guía de Campo. Ed. Corporación Chilena de la Madera, Concepción, Chile. 212 pp.

Saldivia, P. \& G. RoJas. 2006. Valeriana moyanoi Speg. (Valerianaceae), nuevo registro para la flora de Chile. Gayana Botánica 63(2): 183-186.

WAgner, W. H. JR. 1990. Ophioglossaceae. In: K. Kubitzki \& P. S. Green (eds.), The Families and Genera of Vascular Plants, Vol. 1, pp. 193-197. Springer-Verlag, Berlin, Germany.

Recibido: 26.07.11

Aceptado: 02.12.11 